Pathologe 2013 · 34:185

DOI 10.1007/s00292-013-1741-x

Online publiziert: 25. April 2013

(c) Springer-Verlag Berlin Heidelberg 2013

\section{W. Klapper}

Institut für Pathologie, Sektion Hämatopathologie und Lymphknoten Register,

Universitätsklinikum Schleswig-Holstein, Campus Kiel
Wie in allen anderen Bereichen der Tumorpathologie bestimmen zwei Entscheidungen den diagnostischen Prozess:

- Zum einen ist dies die Unterscheidung zwischen einer reaktiv/entzündlichen und einer neoplastischen Veränderung,

- zum anderen - bei neoplastischen Veränderungen - die Festlegung auf eine Tumorentität.

Diese beiden Grenz- und Entscheidungspunkte stellen an den diagnostischen Pathologen auf dem Gebiet der Lymphoproliferationen eine besondere Herausforderung dar.

Die Schwierigkeiten, die alleine die Unterscheidung zwischen einer entzündlichen und einer neoplastischen Veränderung, zum Beispiel im Bereich der Haut bereiten können, basieren meistens nicht auf der Unfähigkeit des Pathologen, sondern auf der Biologie des lymphatischen Systems. Hohe Zellproliferation, unterschiedlichste Zelldifferenzierungen, ortsungebundene Absiedlung und klonale Expansion sind auf der einen Seite physiologische Eigenschaften lymphatischer Zellen, auf der anderen Seite aber auch Kriterien für neoplastische Veränderungen. Hinzu kommt, dass durch neue Erkenntnisse über die Biologie der Erkrankungen und durch die Verfügbarkeit neuer diagnostischer Methoden die Abgrenzung von Lymphomen und entzündlichen Lymphoproliferationen, aber auch von Lymphomentitäten untereinander einem ständigen Wandel unterworfen sind. So wurde zum Beispiel die Grenzziehung zwischen neoplastischen und reaktiven Lymphoproliferationen durch die Beschreibung neuer entzündlicher Proliferationen (z. B. das EBV-assoziierte mukokutane Ulkus) und durch die Identifizierung so genannter früher Läsionen (In-situLymphome) verändert. Darüber hinaus sind Übergänge zwischen Lymphomentitäten in den letzten Jahren durch molekulare Analysen als biologische (z. B. zwischen dem primär mediastinalen großzelligen B-Zell-Lymphom und dem klassischen Hodgkin-Lymphom) oder diagnostische Grauzonen (z. B. zwischen dem diffusen großzelligen B-Zell-Lymphom und dem Burkitt-Lymphom) charakterisiert und neu definiert worden.

Diese neuen Erkenntnisse sind nicht nur für das Verständnis der Pathophysiologie der Erkrankungen von Bedeutung, sondern beeinflussen auch unser diagnostisches Vorgehen.

Das Ziel dieses Themenheftes ist es, die histologischen und molekularen Eigenschaften dieser Grenzbereiche aufzuzeigen und gleichzeitig darzustellen, wie die neuen Erkenntnisse im diagnostischen Alltag umgesetzt werden können. Ich hoffe, dass dabei deutlich wird, dass es nicht immer die Vielzahl oder die Komplexität der immunhistochemischen oder molekularen Analysen ist, die zur "Aufklärung" der Graubereiche führt. Vielmehr ist es meistens die interdisziplinäre Zusammenarbeit des Pathologen mit den behandelnden Ärzten. Die EBV-assoziierten Proliferationen und die frühen Läsionen zeigen uns, dass nicht alles, was unter dem Mikroskop wie ein Lymphom aussieht, sich notwendigerweise klinisch wie ein solches verhält. Ein Pathologe darf den Mut haben, Grauzonen als solche zu benennen, zumal er damit unter Umständen der wahren Biologie des lymphatischen Systems und seiner neoplastischen Veränderungen sehr nahe kommt.

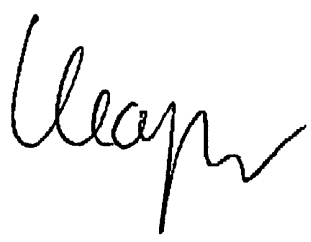

Wolfram Klapper

\section{Korrespondenzadresse}

Prof. Dr. W. Klapper

Institut für Pathologie,

Sektion Hämatopathologie und Lymphknoten

Register, Universitätsklinikum Schleswig-

Holstein, Campus Kiel

Arnold-Heller Straße 3, Haus 14, 24105 Kiel

wklapper@path.uni-kiel.de 